\title{
LÉGISLATION
}

\section{L'électrification des Chemins de fer : La ligne de jonction de l'usine électrogène et de la voie ferrée - Servitudes}

\author{
par Paul Bougault, avocat à la Cour d'appel de Lyon.
}

\begin{abstract}
1 plusieurs reprises, se sont présentées des difficultés au sujet de l'emplacement des lignes électriques reconnues d'utilité publique et par lesquelles l'entrepreneur réclamait le bénéfice des servitudes de l'article 12 de la loi du 15 juin 1906. C'est généralement à l'occasion de l'article 21 de celte loi que les conflits sont nés, c'est-à-dire à l'occasion des conducteurs de courant destinés à réunir une saurce électrogòne à une entreprise de transport en commun déclarée d'utilité publique. Des arrêts de la Cour cé cassation ont été rendus, notamment le 23 juillet 1919 et le 12 juillet 1921. Mais l'arrèt du Conseil d'izlat du 14 janvier 1928 dont on va trouver ci-dessous le texte, est particulièrement intéressant.
\end{abstract}

Principe. - L'article 21 de la loi du 15 juin 1906 permet à toute entreprise déclarée d'utilité publique de réclamer les servitudes d'appui et de passage créées par l'article 12 de la même loi, pour amener le courant aux endroits d'utilisation. Cette faculté, domnée par l'article 21, ne peut-elle ètre octroyée qu'à une entreprise qui a obtenu la déclaration d'utilité publique dans le but de faire usage de ce courant?

Ou bien, cette déclaration ayant été obtenue, mème à l'heure où l'électrification des voies était totalement inconnue, par une Compagnie de chemins de fer, celle-ci peut-elle mettre en œuvre l'article 21, faire approuver un tracé par le Préfet et planter ses pylônes après l'approbation préfectorale ?

Formalités administratives accomplies avec l'approbation du Préfet. - Prétendue violation de la loi résultant de ces formalités. La Compagnie des chemins de fer du Midi a obtenu la déclaration d'utilité publique, en 1865 , pour la création d'un chemin de fer à vapeur (ligne de Montréjeau à Bagnères-de-Luchon). Tout récemment, elle conçut le projet d'électrifier ce tronçon au moyen d'un courant à 60.000 volts amené de Montréjeau (Haute-Garonne) au poste de transformation de Cier-de-Luchon, commune située dans le même département.

Elle avait successivement obtenu :

$1^{0}$ Une décision en date du 24 octobre 1924 , par laquelle le Ministıe des Travaux publics rattachait à la voie ferrée de Montréjeau à Luchon la ligne de transport de courant électrique ;

20 Un arrèté du Préfel du 27 mai 1915 approuvant le tracé que devait suivre, dans diverses communes, cette ligne dite d'électrification, pour rejoindre le réseau de la voie ferrée.

1)eux propriétailes, MM. Dularry et Noguès, formèrent un recours pour excès de pouvoir contre ces deux actes administratifs. Ils voulaient bien admette que l'article 21 de la loi du 15 juin 1906 permet à toute entı eprise déclarée d'utililé publique de réclamer les servitudes d'appui et de passage pour amener le courant sans être tenue de faire déclarer. spécialement d'utilité publique la ligne de transport elle-même, mais ils affirmaient que cette facilité n'est donnée qu’à l'égard d'une œuvre créée pour employer le courant électrique.

Or, disaient-ils, la Compagnie du Midi a une concession qui date de 1865 et n'est relative qu'à la construction d'une voie ferrée. Evidemment, à cette époque, une électrification ne pouvait être envisagée. D'autre part, on a bien formé un projet de concession de distribution d'énergie qui englobe la ligne litigieuse de transport : mais il n'a été mis à l'enquête que le 18 mai 1925. Or, c'est huit jours après (à une date où cette concession n'était encore qu'un projet) que le Préfet a approuvé le tracé proposé par la Compagnie de chemins de fer. Par conséquent, ce tracé ne peut se recommander de la déclaration de 1865, parce qu'elle est très ancienne et ne porte que sur l'établissement d'une voie ferrée, ni alléguer une concession de distribution d'énergie parce qu'elle est simplement projetée : donc, en droit, elle est inexistante.

Réponse du Conseil d'Etat. - La Haute Juridiction a simplement appliqué le texte de l'article 21 de la loi du 15 juin 1906, ainsi conçu : "La déclaration d'utilité publique d'ouvrages à " exécuter par l'Etat, un Département, une Commune, une "Association syndicale, ou par leur concessionnaire, confère à "l'Administration ou au concessionnaire, pour l'établissement "ou le fonctionnement des conducteurs d'énergie employés à "l'exploitation de ces ouvrages, les droits de passage, d'appui, et " d'ébranchage spéciliés à l'article 12 ci-dessus, avec application " des dispositions spéciales édictées à cet effet par les règlements "d'administration publique prévus à l'article 18 " (1).

(1) Il s'agit évidemment du décret du 3 avril 1908 rendu pour l'application de la loi du 15 juin 1906. On sait que ce décret contenait deux articles (36 et 37 ) relatifs aux enquètes pour l'établissement des servitudes d'appui et de passage. Il a été complètement abrogé par le décret du 29 juillet 1927 (Journal officiel, 17 août 1927) qui a refondu tous les textes administratifs réglementant l'application de la loi du 15 juin 1906. Il n'existe donc plus d'intérêt à savoir que les dispositions du décret du 3 avril 1908, en ce qui concerne les concessions de transport d'énergie électrique à haute tension, avaient été modifiées par un décret du 24 avril 
Dans cet article 21, on voit qu'il n'est pas fait la moindre allusion à la date que devrait avoir la déclaration d'utilité publique de l'entreprise qui voudrait pouvoir profiter des facilités relatives aux précieuses servitudes de passage et d'appui.

Il est dit simplement : "La déclaration d'utilité publique " confère à l'Administration ou à son concessionnaire, pour le "fonctionnement des conducteurs employés à l'exploitation de " ces ouvrages, les droits de passage, d'appui, etc... " Aussi, le Conseil d'Etat a déclaré que ni le Ministre, en rattachant le réseau électrique à. la voie ferrée, ni le Préfet en approuvant le tracé de la ligne de transport, n'avait mal interprété la loi, et que le tronçon de raccordement allant de Montréjeau à Cier-deLuchon n'avait pas à être revêtu d'une déclaration spéciale d'utilité publique.

Allant plus loin, le Conseil d'Etat déclare que le bénéfice de cette disposition ne saurait être ni perdu, ni compromis pourla Compagnie des chemins de fer par ce fait que, postérieurement, dans le but d'assurer l'autonomie de cette ligne de transport et de l'englober dans un réseau spécial de distribution, on a entrepris une procédure de déclaration d'utilité publique beaucoup plus générale qui aurait pour but une véritable distribution. Quand on jouit d'un droit précis, il serait peu indiqué que l'on fût dépossédé de ce droit par le seul fait que, plus tard, il se présentera une extension du fait primitif.

\section{Texte de l'arrêt du 14 janvier 1928. - Le Conseil d'Etat,}

Vu la requète présentée par les sieurs Dubarry et Noguès, propriétaires, demeurant à Loures-Barousse (Hautes-Pyrénées), ladite requête enregistrée au Secrétariat du Contentieux du Conseil d'Etat le 15 juin 1925 et tendant à ce qu'il plaise au Conseil annuler, pour excès de pouvoir, un arrêté en date du 27 mai 1925, par lequel le Préfet du département des Hautes-Pyrénées a approuvé le projet de tracé d'une ligne de transport d'énergie électrique de 60.000 volts de Montréjeau à Cier-de-Luchon dans sa traversée de la Commune de Loures et, en tant que de besoin, une décision du Ministre des Travaux publics, en date du 24 octobre 1924, déclarant que cette ligne de transport électrique était rattachée à la ligne de chemin de fer de Montréjeau à Luchon;

Ce faire, attendu que l'arrêté approuvant le tracé, en déterminant les parcelles sur lesquelles le concessionnaire privilégié pourra exercer les servitudes d'utilité publique auxquelles la déclaration d'utilité publique ne lui donnait qu'un droit éventuel et indéterminé, fait directement grief à l'assujetti ; que celui-ci n'a d'autre voie pour libérer son fonds de la servitude de la loi de 1906 que le recours en annulation pour excès de pouvoir;

Attendu que le Préfet n'a ouvert l'enquête sur l'avant-projet de concession dans la Commune de Loures que par arrêté du 18 mai 1925 ; que, ainsi, à la date de l'arrêté attaqué, le réseau

1923 (Journal officiel, 29 avril 1923) et que ce dernier décret avait été lui-même modifié par un autre en date du 14 octobre 1924 (Journal officiel, 18 octobre 1924). Indiquons encore que, après la loi du 27 février 1925, modifiant le régime juridique des autorisations de voirie, l'article 4 du décret du 3 avril 1908 avait été modifié, en ce qui concerne la présentation des demandes d'autorisation, par décret du $27^{\circ}$ septembre 1926 (Journal officiel, 8 octobre 1926). Cette modification est reproduite dans le décret précité du 29 juillet 1927 qui se suffit donc à lui-même. En résumé, les deux textes administratifs réglementant l'application de la loi, sont aujourd'hui le décret du 27 septembre 1926 en ce qui concerne les autorisations de voirie, et le décret du 29 juillet 1927 qui a une portée très générale, pour toutes les formalités à accomplir. électrique à haute tension dont fait partie la ligne de Montréjeau à Cier-de-Luchon n'était ni concédé ni déclaré d'utilité publique ; que, par suite, tout arrêté approuvant le tracé est illégal ;

Que la Compagnie du Midi a procédé en suivant les prescriptions légales à rebours; qu'elle a commencé par prendre possession effective des parcelles convoitées, y a fait planter des pylònes, et mème poser les fils de la ligne dont elle escompte la concession, avant d'avoir rempli les formalités administratives préalablement requises ;

Que, si l'article 21 de la loi du 15 juin 1906 accorde à l'Etat ou aux Compagnies de chemins de fer le bénéfice des dispositions de l'article 12 de la même loi, sans nouvelle déclaration d'utilité publique, pour les lignes électriques devant servir à l'exploitation d'ouvrages d'utilité publique, ce texle ne peut s'appliquer qu'à des travaux publics pour l'exploitation desquels la concession avait prévu l'emploi de l'énergie électrique; que l'utilité publique d'une ligne de 60.000 volts ne pouvait être impliquée et sous-entendue en 1865 dans la déclaration d'utilité publique d'un chemin de fer à la vapeur tel que la voie ferrée de Montréjeau à Bagnères-de-Luchon ; que les deux lignes n'ont ni la mème nature ni le mème tracé; qu'il est inexact en fail de soutenir que la ligne électrique projetée servira au lonctionnement du chemin de fer de Montréjeau à Luchon ; que la ligne à haute tension n'est pas utilisable pour la traction des trains qui se fait par une ligne à basse tension de 1.500 volts ;

Que le Ministre des Travaux publics n'était pas qualifié pour faire la déclaration de rattachement du 24 octobre 1924 ; que, au surplus, si cette déclaration avait quelque fondement sérieux, on ne comprendrait pas que la Compagnie du Midi ait demande la déclaration d'utilité publique de cette ligne, que le Ministre ait ordonné l'enquête sur celte utilité publique et que le Préfel y fasse actuellement procéder :

\section{Vu les décisions attaquées;}

Vu les observation présentées par la Compagnie des chemins de fer du Midi en réponse à la communication qui lui a été donnée du pourvoi, lesdites observations enregistrées comme ci-dessus, le 25 août 1925, et tendant au rejet dudit pourvoi, par les motifs :

Que le sieur Noguès lui ayant, postérieurement au dépôt de la requête, accordé le droit d'installer, sur sa propriété, la ligne de transport d'énergie en question, n'a plus aucun intérêt à poursuivre son recours;

Que, en vertu de l'article 21 de la loi du 15 juin 1906, il a pu être procédé immédiatement aux enquêtes de servitudes, sans autre formalité préalable que la décision ministérielle du 3 décembre 1924 déclarant la ligne électrique projetéc travail complémentaire du chemin de fer; que les lignes électriques à 60.000 volts de la Compagnie du Midi sont avant tout construites pour l'électrification de la voie ferrée;

Que si la ligne de transport d'énergie Montréjeau-Cier-de-Luchon, construite avant tout pour l'électrification du réseau, a été comprise dans un réseau de distribution susceptible d'être détaché plus tard de la concession de chemin de fer et pour lequel la procédure de concession a été ouverte, ce fait, conséquence du but poursuivi, qui est l'indépendance ultérieure des lignes de transport d'énergie vis-à-vis des concessions de chemins de fer, n'a pour effet de vicier la procédure toute différente prévue par l'article 21 de la loi du 15 juin 1906 ;

Vu les observations présentées par le Ministre des Travaux publics en réponse à la communication qui lui a été donnée du 
pourvoi, lesdites observations enregistrées comme ci-dessus, le 15 octobre 1925, et tendant au rejet dudit pourvoi par les motifs : que l'examen des travaux préparatoires de la loi de 1906 démontre que le texte de l'article 21 a été inséré dans la loi à l'intention spéciale des tramways ou des chemins de fer qui auraient à s'électrifier; qu'il devait s'appliquer en l'espèce; que, rien ne permet de déduire de la rédaction très générale dudit article 21 qu'il ne s'applique que si l'électrification des ouvrages a été envisagée dès l'origine, si les ouvrages et la ligne de transport sont de même nature, ont le même tracé ; que si, enfin, les lignes d'alimentation sont à la tension d'utilisation des moteurs; que, dans ces conditions, le Ministre des Travaux publics était fondé à reconnaître à la Compagnie des chemins de fer du Midi le droit d'invoquer l'article 21 de la loi du 15 juin 1906 et le Préfet des Hautes-Pyrénées à approuver ensuite le tracé de cette ligne, après exécution des enquêtes prévues par les articles 12, 18 et 21;

$\mathrm{Vu}$ les autres pièces produites et jointes au dossier ;

Vu la loi du 15 juin 1906 ;

Vu les lois des 7-14 octobre 1790 et 24 mai 1872 ;

Ouï M. Gélinet, Auditeur, en son rapport;

Ouï M. Rouchon-Mazerat, Maître des Requêtes, Commissaire du Gouvernement, en ses conclusions;

Sans qu'il soit besoin de statuer sur la fin de non-recevoir opposéc à l'un des requérants par le Ministre des Travaux publics ;

Considérant que, par les décisions attaquées, le Ministre des Travaux publics, d'une part, a reconnu à la Compagnie des chemins de fer du Midi, pour les travaux de construction de la ligne de transport d'énergie électrique de Montréjeau à Cier-deLuchon, envisagés comme complémentaires de ceux de la voie ferrée, le droit de se prévaloir de l'article 21 de la loi du 15 juin 1906, et le Préfet du département des Hautes-Pyrénées, d'autre part, a approuvé le tracé de ladite ligne dans sa traversée de la Commune de Loures;

Considérant que, pour demander l'annulation de ces deux décisions qui ont eu pour effet de rendie applicables dans ladite Commune les servitudes prévues par l'article 12 de la loi du
15 juin 1906, les sieurs Dubarry et Noguès soutiennent que les travaux dont s'agit ne pouvaient être regardés, à raison de leur nature et de leur tracé différent de celui de la voie ferrée qui les utilise, comme se rattachant à la concession de la ligne de Montréjeau à Bagnères-de-Luchon et compris comme tels, dans la déclaration d'utilité publique prononcée par décret du 14 décembre 1865, lors de l'établissement de ladite ligne ;

Considérant que les distributions d'énergie électrique desservant les chemins de fer et les tramways sont au nombre de celles que régit la loi du 15 juin 1906 ; mais que, aux termes de l'article 21 de ladite loi, les ouvrages exécutés par l'Etat, un Département, une Commune, ou par leurs concessionnaires, lorsqu'ils bénéficient déjà d'une déclaration d'utilité publique, jouissent des droits de passage, d'appui et d'ébranchage spécifiés à l'article 12 ; que, si cette disposition législative n'a pas pour effet d'affranchir le concessionnaire de l'observation des formalités prescrites par les articles 12,18 et 21 , elle les dispense de solliciter de l'Administration toute nouvelle concession pour profiter des avantages ci-dessus rappelés ;

Considérant que, si, à la vérité, il a paru opportun aux Pouvoirs publics d'engager cependant la procédure de la déclaration d'utilité publique du réseau électrique comprenant la ligne de transport d'énergie de Montréjeau à Luchon en vue d'assurer son autonomie et de l'utiliser éventuellement à d'autres fins que la seule exploitation de la voie ferrée, cette circonstance n'était pas de nature à priver la Compagnie des chemins de fer du Midi des droits ci-dessus définis qu'elle tenait tant de sa concession primitive que de l'article 21 de la loi du 15 juin 1906 ;

Considérant qu'il résulte de ce qui précède que les décisions attaquées ne sont pas entachées d'excès de pouvoir et que la requête des sieurs Dubarry et Noguès doit être rejetée ;

Décide :

Article $1^{\mathrm{er}}$. - La requête susvisée des sieurs Dubarry et Noguès est rejetée.

Article 2. - Expédition de la présente décision sera transmise au Ministre des Travaux publics. 